\title{
RIGOROUS DERIVATION OF THE HYDRODYNAMICAL EQUATIONS FOR ROTATING SUPERFLUIDS
}

\author{
HAILIANG LIU AND CHRISTOF SPARBER
}

\begin{abstract}
Using a modified WKB approach, we present a rigorous semiclassical analysis for solutions of nonlinear Schrödinger equations with rotational forcing. This yields a rigorous justification for the hydrodynamical system of rotating superfluids. In particular it is shown that global-in-time semiclassical convergence holds whenever the limiting hydrodynamical system has global smooth solutions and we also discuss the semi-classical dynamics of several physical quantities describing rotating superfluids.
\end{abstract}

\section{Physical Motivation}

Bose-Einstein condensates (BECs) play an outstanding role in present-day physics, cf. 22 for a general introduction. Understanding and controlling their behavior is of great fundamental importance and essential for novel applications. A particular focus of interest is on dynamical phenomena related to superfluidity and the creation of quantized vortices, see, e.g., [1, 12, 15, 16. To this end the typical experimental set-up is based on a trapping potential subject to a rotational forcing. The mathematical description of this system is then usually given by the celebrated Gross-Pitaevskii equation (GPE), a mean field model for the macroscopic wave function of the condensate (see [17] for a rigorous derivation). In the rotating reference frame we are consequently led to

$$
\mathrm{i} \hbar \partial_{t} \psi=-\frac{\hbar^{2}}{2 m} \Delta \psi+V_{0}(x) \psi+\kappa|\psi|^{2} \psi+\mathrm{i} \Omega_{0} x^{\perp} \cdot \nabla_{x} \psi,
$$

with a nonlinear coupling constant $\kappa=N 4 \pi \hbar a / m$, where $N$ is the number of particles forming the condensate and $m, a \in \mathbb{R}$ respectively denote their corresponding mass and scattering length. In the context of BECs the potential $V$ is usually assumed to be a harmonic oscillator confinement, i.e. $V_{0}(x)=\frac{1}{2} m \omega_{0}^{2}|x|^{2}$, for some $\omega_{0} \in \mathbb{R}^{d}$. In (1.1) we also write $x^{\perp}=\left(x_{2},-x_{1}, 0\right)^{\top}$ in $d=3$ spatial dimensions and analogously $x^{\perp}=\left(x_{2},-x_{1}\right)^{\top}$ for $d=2$. The latter case is motivated by recent experiments for BECs which are strongly confined in one or two directions. Thus $x^{\perp} \cdot \nabla_{x}$ can be interpreted as the negative $x_{3}$-component of the quantum mechanical angular momentum operator $L=x \times\left(-\mathrm{i} \nabla_{x}\right)$ and $\Omega_{0}$ is the corresponding angular velocity.

In the so-called Thomas-Fermi limit, which typically applies for large systems, the dynamics is presumably well described by the hydrodynamical equations for rotating

\footnotetext{
Key words and phrases. Semi-classical asympotics, nonlinear Schrödinger equation, BoseEinstein condensates, rotational superfluids, Thomas-Fermi limit.

C. S. has been supported by the APART grant of the Austrian Academy of Sciences. H. L. was partially supported by the National Science Foundation under Grant DMS05-05975.
} 
superfluids [2, 23, 24, i.e.

$$
\left\{\begin{array}{l}
\partial_{t} \rho+\nabla_{x} \cdot\left(\rho\left(\mathbf{v}-\Omega_{0} x^{\perp}\right)\right)=0 \\
\partial_{t} \mathbf{v}+\nabla_{x}\left(\frac{|\mathbf{v}|^{2}}{2}-\Omega_{0} x^{\perp} \cdot \mathbf{v}+V_{0}+\rho\right)=0,
\end{array}\right.
$$

where $\rho:=|\psi|^{2}$ denotes the particle density and $\mathbf{v}$ the corresponding superfluid velocity defined by

$$
\mathbf{v}:=\frac{\hbar}{m} \frac{\operatorname{Im}\left(\bar{\psi} \nabla_{x} \psi\right)}{|\psi|^{2}}
$$

The passage from (1.1) to (1.2) is usually explained by using the classical Madelung transformation of the wave function, where one writes

$$
\psi(t, x)=\sqrt{\rho(t, x)} \exp (\mathrm{i} \Phi(t, x) / \hbar),
$$

and consequently identifies $\mathbf{v}:=\nabla_{x} \Phi$. Formally plugging the ansatz (1.3) into (1.1), separating real and imaginary parts, and discarding terms $\propto \hbar^{2}$ yields (1.2). This asymptotic regime is particularly interesting for numerical simulations, $c f$. 4, 9], and we also refer to [18, 11] for an extensive review of such dispersive limits. However, the representation (1.3) makes no sense at vacuum points, where $\rho=0$. Indeed the system (1.2) degenerates at such points and is therefore only weakly hyperbolic (see, e.g., [18).

In the present work we shall rigorously prove that (1.7) approximates (1.1) in a certain sense and moreover draw some further conclusions from that. To this end we rescale equation (1.1), as described in [5, 20, into its dimensionless form. This yields

$$
\mathrm{i} \epsilon \partial_{t} \psi^{\epsilon}=-\frac{\epsilon^{2}}{2} \Delta \psi^{\epsilon}+V(x) \psi^{\epsilon}+\epsilon \delta^{5 / 2}\left|\psi^{\epsilon}\right|^{2} \psi^{\epsilon}+\mathrm{i} \epsilon \Omega x^{\perp} \cdot \nabla_{x} \psi^{\epsilon},
$$

with $\epsilon=\hbar /\left(m L^{2}\right)$ and $\delta=4 \pi a N / a_{0}$, where $L$ denotes the characteristic length of the condensate and $a_{0}=\sqrt{\hbar /\left(m \omega_{0}\right)}$ is the ground state length of the harmonic oscillator potential $V(x)$. From now on $\Omega$ and $V(x)$ denote (rescaled) dimensionless quantities. The particular choice $\left|\epsilon \delta^{5 / 2}\right|=\mathcal{O}(1)$, which yields $a_{0} \ll L$ and thus $\epsilon=\left(a_{0} / 4 \pi|a| N\right)^{1 / 5} \ll 1$, corresponds to the Thomas-Fermi regime of stronginteractions, $c f$. 5 for more details. Note however that our scaling is different from the one used in [1, 2, 13].

We consequently study the following semi-classically scaled nonlinear Schrödinger equation (NLS) with rotational forcing

$$
\left\{\begin{array}{l}
\mathrm{i} \epsilon \partial_{t} \psi^{\epsilon}=-\frac{\epsilon^{2}}{2} \Delta \psi^{\epsilon}+V(x) \psi^{\epsilon}+f\left(\left|\psi^{\epsilon}\right|^{2}\right) \psi^{\epsilon}+\mathrm{i} \epsilon \Omega x^{\perp} \cdot \nabla_{x} \psi^{\epsilon} \\
\left.\psi^{\epsilon}\right|_{t=0}=\psi_{\mathrm{in}}^{\epsilon}(x),
\end{array}\right.
$$

where $t \in \mathbb{R}, x \in \mathbb{R}^{d}$, for $d=2,3$, and $\Omega \geq 0$, some given constant (independent of $\epsilon \ll 1)$. The energy functional associated to (1.5) reads

$$
E\left(\psi^{\epsilon}\right)=\int_{\mathbb{R}^{d}} \frac{\epsilon^{2}}{2}\left|\nabla_{x} \psi^{\epsilon}\right|^{2}+\left(V(x)+F\left(\left|\psi^{\epsilon}\right|^{2}\right)\right)\left|\psi^{\epsilon}\right|^{2}+\mathrm{i} \epsilon \Omega \overline{\psi^{\epsilon}} x^{\perp} \cdot \nabla_{x} \psi^{\epsilon} \mathrm{d} x,
$$

where $F$ is the primitive of $f$. From now on we impose the following assumptions.

Assumption 1. It holds:

- The nonlinearity satisfies $f \in C^{\infty}(\mathbb{R})$ such that $f^{\prime}>0$.

- The potential $V$ is of the form $V(x)=\frac{1}{2}|\omega \cdot x|^{2}$, with $\omega \in \mathbb{R}^{d}$. 
The superfluid equations corresponding to the NLS (1.5) are thus given by

$$
\left\{\begin{array}{l}
\partial_{t} \rho+\nabla_{x} \cdot\left(\rho\left(\mathbf{v}-\Omega x^{\perp}\right)\right)=0 \\
\partial_{t} \mathbf{v}+\nabla_{x}\left(\frac{|\mathbf{v}|^{2}}{2}-\Omega x^{\perp} \cdot \mathbf{v}+V+f(\rho)\right)=0 .
\end{array}\right.
$$

We expect the system (1.7) to be a valid description of solutions to (1.5) in the limit $\epsilon \rightarrow 0$. In order to prove this rigorously, we will heavily rely on semi-classical expansion techniques first developed by Grenier [19] and later extended by Carles in [10. The latter work in particular treats the case of a harmonic confinement and nonlinearities of different strength (see also [3] for an extension to higher order nonlinearities and [14] for results on the derivative NLS). We can thus focus on the role played by the rotational forcing. Indeed the motivation for our work is threefold: First, we aim to generalize the results of [10] to the case where a rotational term is included and consequently give a rigorous justification of (1.7). Second we strengthen these results a bit in the sense of Corollary 3.1 below. Finally we aim to describe the dynamical features of rotational BECs from the semi-classical point of view, as given in Theorem 4.1

\section{The MODIFIED WKB-APPROACH}

As noticed in 10, 19, the classical Madelung transformation (1.3) is not well suited to rigorously derive the semi-classical asymptotics of $\psi^{\epsilon}$. Rather one is led to consider a modified version of it. To this end one writes the exact solution to (1.5) in the form

$$
\psi^{\epsilon}(t, x)=a^{\epsilon}(t, x) \mathrm{e}^{\mathrm{i} \Phi^{\epsilon}(t, x) / \epsilon}
$$

where from now on the "amplitude" $a^{\epsilon}$ is allowed to be complex-valued. Moreover $a^{\epsilon}$ as well as the (real-valued) phase $\Phi^{\epsilon}$ are assumed to admit an asymptotic expansion of the form

$$
a^{\epsilon} \sim a+\epsilon a_{1}+\epsilon^{2} a_{2}+\cdots, \quad \Phi^{\epsilon} \sim \Phi+\epsilon \Phi_{1}+\epsilon^{2} \Phi_{2}+\cdots .
$$

Since $a^{\epsilon}$ is complex valued, the phase $\Phi^{\epsilon}$ can be seen as an additional degree of freedom introduced as a multiple scales representation for $\psi^{\epsilon}$. In any case, the ansatz (2.1) should not be confused with (1.3) and in particular it has nothing to do with a rewriting of $\psi^{\epsilon}$ into polar coordinates. The main gain of this modified WKB-approach is that it yields a separation of scales within the appearing fast, i.e. $\epsilon$-oscillatory, phases and slowly varying phases, which eventually can be included in the (complex-valued) amplitudes.

An analysis based on WKB-type methods necessary requires admissible initial data. To this end we introduce the following definition: A function $f \in C^{\infty}\left(\mathbb{R}^{d}\right)$ is said to be sub-quadratic, if

$$
\forall \alpha \in \mathbb{N}^{d},|\alpha| \geq 2: \sup _{x \in \mathbb{R}^{d}}\left|\partial_{x}^{\alpha} f\right|<\infty .
$$

We consequently impose:

Assumption 2. The initial data is of the form

$$
\psi_{\text {in }}^{\epsilon}(x)=a_{\text {in }}^{\epsilon}(x) \mathrm{e}^{\mathrm{i} \Phi_{\text {in }}(x) / \epsilon} .
$$

Here $a_{\text {in }}^{\epsilon}$ is complex-valued and admits an asymptotic expansion in $\epsilon$ whereas $\Phi_{\text {in }}(x)$ is $\epsilon$-independent, real-valued, and sub-quadratic. 
It is important to note that Assumption 2 imposes a particular $\epsilon$-oscillatory structure on the initial data but does not yield any problems at vacuum points since $a_{\text {in }}^{\epsilon}$ is allowed to be complex-valued.

Remark 2.1. In particular we are free to choose, say in $d=2$ spatial dimensions, an initial data of the form: $\Phi_{\text {in }}(x)=0, a_{\text {in }}^{\epsilon}(x)=\chi(r) \mathrm{e}^{\mathrm{i} m \theta}$, where we have used polar coordinates. Here $m \in \mathbb{N}_{0}$ is the so-called winding number. For $m \neq 0$ and $\chi$ appropriately chosen (see, e.g., [4, 8, 6]) this allows for so-called vortex states as initial data. We also note that we could allow for more general initial phase function $\Phi_{\text {in }}^{\epsilon}$ which admit an asymptotic expansion in powers of $\epsilon$ (analogously to $\left.a_{\text {in }}^{\epsilon}\right)$. For simplicity we do nit treat this case though.

Upon substituting (2.1) into the NLS (1.5), we have the freedom to split the appearing terms into

$$
\left\{\begin{array}{l}
\partial_{t} \Phi^{\epsilon}+\frac{1}{2}\left|\nabla_{x} \Phi^{\epsilon}\right|^{2}+V(x)-\Omega x^{\perp} \cdot \nabla_{x} \Phi^{\epsilon}+f\left(\left|a^{\epsilon}\right|^{2}\right)=0, \\
\partial_{t} a^{\epsilon}+\nabla_{x} \Phi^{\epsilon} \cdot \nabla_{x} a^{\epsilon}+\frac{1}{2} a^{\epsilon} \Delta \Phi^{\epsilon}-\Omega x^{\perp} \cdot \nabla_{x} a^{\epsilon}=\frac{\mathrm{i} \epsilon}{2} \Delta a^{\epsilon} .
\end{array}\right.
$$

This system is equivalent to the nonlinear Schrödinger equation (1.5). In particular it does no longer represent a splitting into real and imaginary parts (since $a^{\epsilon}$ is complex-valued), in contrast Madelung's original approach. Moreover the system (2.5) is seen to be perturbed by a term which is "only" $\mathcal{O}(\epsilon)$ (since the term $\propto \Delta a$ now appears in the equation for the amplitude instead of the one for the the phase). As $\epsilon \rightarrow 0$, the corresponding limiting WKB system is then (formally) found to be

$$
\left\{\begin{array}{l}
\partial_{t} \Phi+\frac{1}{2}\left|\nabla_{x} \Phi\right|^{2}+V(x)-\Omega x^{\perp} \cdot \nabla_{x} \Phi+f\left(|a|^{2}\right)=0, \\
\partial_{t} a+\nabla_{x} \Phi \cdot \nabla_{x} a+\frac{1}{2} a \Delta \Phi-\Omega x^{\perp} \cdot \nabla_{x} a=0 .
\end{array}\right.
$$

From here, setting $\rho=|a|^{2}$ and $\mathbf{v}=\nabla_{x} \Phi$, one (again formally) obtains a hydrodynamical equations of superfluids (1.7). Indeed we shall prove below that (2.6) and (1.7) are in a certain sense equivalent.

Remark 2.2. From the point of view of geometrical optics, the above given limit corresponds to the supercritical case, cf. [10, 3, 11] for more details. Note however that a rotational forcing is neither considered in [10, 3, nor in 18. Also note that in the case of a linear Schrödinger equation the corresponding system (2.6) would be decoupled since the first equation would be replaced by the classical rotational Hamilton-Jacobi equation (HJ)

$$
\partial_{t} S+\frac{1}{2}\left|\nabla_{x} S\right|^{2}+V(x)-\Omega x^{\perp} \cdot \nabla_{x} S=0 .
$$

Equations of the form (2.7) have been extensively studied in [20], where several qualitative properties for the corresponding solutions are established.

As a first step in our analysis we shall show that the usual hydrodynamical system (1.7) and the WKB system (2.6) admit smooth solutions on the same time-intervall.

Lemma 2.1 (Equivalence). Let $T$ be the maximal time of existence for a smooth solution $(\rho, \mathbf{v})$, with $\rho \geq 0$, of the hydrodynamical system (1.7) and let $T^{*}$ be the maximal existence-time of a smooth solution $(a, \Phi)$ of (2.6). Then we have $T=T^{*}$. 
Proof. We define $(\alpha, \beta, \mathbf{v}):=\left(\operatorname{Re} a, \operatorname{Im} a, \nabla_{x} \Phi\right)$ and rewrite (2.6) in the following form

$$
\left\{\begin{array}{l}
\partial_{t} \alpha+\mathbf{v} \cdot \nabla_{x} \alpha+\frac{\alpha}{2} \nabla_{x} \cdot \mathbf{v}-\Omega x^{\perp} \cdot \nabla_{x} \alpha=0, \\
\partial_{t} \beta+\mathbf{v} \cdot \nabla_{x} \beta+\frac{\beta}{2} \nabla_{x} \cdot \mathbf{v}-\Omega x^{\perp} \cdot \nabla_{x} \beta=0, \\
\partial_{t} \mathbf{v}+\nabla_{x}\left(\frac{|\mathbf{v}|^{2}}{2}-\Omega x^{\perp} \cdot \mathbf{v}+V+f\left(\alpha^{2}+\beta^{2}\right)\right)=0 .
\end{array}\right.
$$

Assume that $(\alpha, \beta, \mathbf{v})$ is a smooth solution of the modified WKB system (2.8) for $t \in\left[0, T^{*}\right)$. Then $\eta=\alpha^{2}+\beta^{2}$ and $\mathbf{v}=\nabla_{x} \Phi$ are smooth solutions of (1.7). By uniqueness it follows that $(\eta, \mathbf{v})=(\rho, \mathbf{v})$ for $t \leq T^{*}$ and hence $T^{*} \leq T$.

Conversely, assume that $(\rho, \mathbf{v})$ is the smooth solution of the hydrodynamic system (1.7) for $t \in[0, T]$, subject to initial data $\left(\rho_{\text {in }} \geq 0, \mathbf{v}_{\text {in }}\right)$ such that $\rho_{\text {in }}=\alpha_{\text {in }}^{2}+\beta_{\text {in }}^{2}$. By assumption the velocity $\mathbf{v}$ is smooth, one thus obtains smooth $a$ and $b$ from the first two transport equations in (2.8), subject to initial conditions $\left(\alpha_{\text {in }}, \beta_{\text {in }}\right)^{\top}$. A combination of the two equations for $\alpha$ and $\beta$ gives

$$
\partial_{t}\left(\alpha^{2}+\beta^{2}\right)+\mathbf{v} \cdot \nabla_{x}\left(\alpha^{2}+\beta^{2}\right)=-\left(\alpha^{2}+\beta^{2}\right) \nabla_{x} \cdot \mathbf{v}+\Omega x^{\perp} \cdot \nabla_{x}\left(\alpha^{2}+\beta^{2}\right) .
$$

Subtracting this from the equation for $\rho$ in (1.7), we find that $\tilde{\rho}:=\rho-\left(\alpha^{2}+\beta^{2}\right)$ solves a transport equation

$$
\partial_{t} \tilde{\rho}+\nabla_{x} \cdot(\mathbf{v} \tilde{\rho})=\Omega x^{\perp} \cdot \nabla_{x} \tilde{\rho},
$$

with $\left.\tilde{\rho}\right|_{t=0}=0$, and hence $\tilde{\rho}(t, x) \equiv 0$, i.e. $\rho=\alpha^{2}+\beta^{2}$. This shows that $(\rho, \mathbf{v})$ is also the smooth solution of the hydrodynamic system (1.7) for $t \in\left[0, T^{*}\right]$, hence $T \leq T^{*}$. In summary this yields $T=T^{*}$ for the solutions of (2.6) and (2.8). To get back to $\Phi$ itself, we first note that in the equation

$$
\partial_{t} \Phi+\frac{1}{2}\left|\nabla_{x} \Phi\right|^{2}+V(x)-\Omega x^{\perp} \cdot \nabla_{x} \Phi+f\left(|a|^{2}\right)=0
$$

all terms are uniquely determined by (2.8) except for $\partial_{t} \Phi$. Imposing $\left.\Phi\right|_{t=0}=\Phi_{\mathrm{in}}$, such that $\mathbf{v}_{\text {in }}=\nabla_{x} \Phi_{\text {in }}$, and setting

$$
\Phi(t, x)=\Phi_{\text {in }}(x)+\int_{0}^{t}\left(\frac{1}{2}|\mathbf{v}(\tau, x)|^{2}+|a(\tau, x)|^{2}+V(x)-\Omega x^{\perp} \cdot \mathbf{v}(\tau, x)\right) \mathrm{d} \tau,
$$

we infer that $\partial_{t}\left(\nabla_{x} \Phi-\mathbf{v}\right)=\nabla_{x} \partial_{t} \Phi-\mathbf{v}=0$, hence $\mathbf{v}=\nabla_{x} \Phi$. This then fully determines $\Phi$ on $[0, T]$.

Having established the equivalence between the limiting systems (1.7) and (2.6), we can now focus on deriving rigorously (2.6) from (2.5) (or, equivalently, the NLS (1.5)).

\section{The ClASSICAL Limit}

From now on we shall mainly consider the WKB system (2.6) in the form (2.8) which allows for a treatment in the sense of hyperbolic systems. In the corresponding analysis, the rotational HJ equation (2.7) becomes important. As a preparatory step we shall therefore study the Cauchy problem

$$
\left\{\begin{array}{l}
\partial_{t} S+\frac{1}{2}\left|\nabla_{x} S\right|^{2}+V(x)-\Omega x^{\perp} \cdot \nabla_{x} S=0, \\
\left.S\right|_{t=0}=S_{\text {in }}(x),
\end{array}\right.
$$

The following result will be used throughout this work. 
Lemma 3.1. Let Assumption 1 hold. If $S_{\mathrm{in}}(x) \in C^{\infty}\left(\mathbb{R}^{d}\right)$ is sub-quadratic, then there exists a $\tau>0$ such that (2.7) admits a unique smooth solution for $t \in[0, \tau)$. Moreover, the phase $S(t, x)$ remains sub-quadratic in $x$, for all $t \in[0, \tau)$.

As pointed out in [10, the sub-quadratic assumption for the initial phase is sharp for solving (2.7) globally in space even in the presence of no rotational force. Concerning the existence of global-in-time smooth solutions we refer to [20].

Proof. The Hamiltonian corresponding to 3.1 is

$$
H=\frac{1}{2}|p|^{2}+V(x)-\Omega x^{\perp} \cdot p .
$$

and thus the corresponding Hamiltonian flow is governed by

$$
\left\{\begin{array}{l}
\dot{x}=p-\Omega x^{\perp},\left.\quad x\right|_{t=0}=x_{0}, \\
\dot{p}=-\omega x-\Omega p^{\perp},\left.\quad p\right|_{t=0}=\nabla_{x} S_{\text {in }}\left(x_{0}\right) .
\end{array}\right.
$$

Standard ODE theory implies that there exists a unique solution for $t \in[0, \tau]$, in which the map $x=x\left(t, x_{0}\right)$ is well defined, satisfying

$$
\operatorname{det}(\Gamma)>0, \quad \Gamma:=\frac{\partial}{\partial x_{0}} x\left(t, x_{0}\right) .
$$

Existence of smooth solutions is therefore guaranteed and we now have to prove that $S$ remains sub-quadratic for $t \in[0, \tau]$. From [20] we infer that the phase gradient $u=\nabla_{x} S$ satisfies

$$
\partial_{t} u+\left(u-\Omega x^{\perp}\right) \cdot \nabla_{x} u=-\omega x-\Omega u^{\perp} .
$$

Further, along the particle path induced via $\dot{x}=u-\Omega x^{\perp}$ the Hessian of the phase function $\Sigma:=D_{x}^{2} S(t, x)$ solves a matrix ODE

$$
D_{t} \Sigma+\Sigma^{2}=-\omega \mathbf{I}_{d}
$$

where we shortly denote

$$
D_{t}:=\partial_{t}+\left(U-\Omega x^{\perp}\right) \cdot \nabla_{x}
$$

This shows that $\Sigma$ remains uniformly bounded in terms of $x_{0} \in \mathbb{R}^{d}$. Finally we show that the existence time $\tau$ does not shrink as $x$ varies over $\mathbb{R}^{d}$. Differentiation of $\dot{x}=u-\Omega x^{\perp}$ in terms of $x_{0}$ yields

$$
D_{t} \Gamma=(\Sigma-\Omega J) \Gamma, \quad J=\left(\begin{array}{ccc}
0 & 1 & 0 \\
-1 & 0 & 0 \\
0 & 0 & 0
\end{array}\right)
$$

for $d=3$. (The expression for $J$ in the case $d=2$ is obvious.) This gives

$$
\operatorname{det}(\Gamma)=\exp \left(\int_{0}^{t} \operatorname{Tr}(\Sigma-\Omega J) \mathrm{d} \tau\right)=\exp \left(\int_{0}^{t} \operatorname{Tr} \Sigma \mathrm{d} \tau\right),
$$

where $\operatorname{Tr}$ denotes the standard trace map. The fact that $\operatorname{det}(\Gamma) \operatorname{does}$ not depend on $x_{0}$ explicitly implies that $\Sigma$ is uniformly bounded in $x_{0} \in \mathbb{R}^{d}$.

In order to prove the local-in-time existence for the system (2.5), we follow the strategy in [10] and decompose the phase $\Phi^{\epsilon}$ into

$$
\Phi^{\epsilon}=\varphi^{\epsilon}+S
$$


where $S$ is the smooth, sub-quadratic phase function guaranteed by Lemma 3.1. In terms of $\varphi^{\epsilon}$ and $a^{\epsilon}$ the system (2.5) becomes

$$
\left\{\begin{array}{l}
\partial_{t} \varphi^{\epsilon}+\nabla_{x} S \cdot \nabla_{x} \varphi^{\epsilon}+\frac{1}{2}\left|\nabla_{x} \varphi^{\epsilon}\right|^{2}-\Omega x^{\perp} \cdot \nabla_{x} \varphi^{\epsilon}+f\left(\left|a^{\epsilon}\right|^{2}\right)=0 \\
\partial_{t} a^{\epsilon}+\nabla_{x}\left(S+\varphi^{\epsilon}\right) \cdot \nabla_{x} a^{\epsilon}+\frac{a^{\epsilon}}{2} \Delta\left(S+\varphi^{\epsilon}\right)-\Omega x^{\perp} \cdot \nabla_{x} a^{\epsilon}=\frac{\mathrm{i} \epsilon}{2} \Delta_{x} a^{\epsilon} .
\end{array}\right.
$$

Note that this set of equations is still equivalent to the nonlinear Schrödinger equation (1.5). The reason for decomposing $\Phi^{\epsilon}$ via (3.3) is rather technical and due to the inclusion of the potential $V$ and the rotational term.

For notational convenience we further introduce

$$
U^{\epsilon}:=\left(\operatorname{Re} a^{\epsilon}, \operatorname{Im} a^{\epsilon}, \partial_{x_{1}} \varphi^{\epsilon}, \ldots, \partial_{x_{d}} \varphi^{\epsilon}\right)^{\top},
$$

where $a^{\epsilon}$ and $\varphi^{\epsilon}$ satisfy (3.4) (an analogous notation is used for the corresponding initial data). Moreover, we shall frequently use the notation

$$
\mathcal{N}\left[U^{\epsilon}(t]\right):=\left\|U^{\epsilon}(t)\right\|_{s}+\left\||x| U^{\epsilon}(t)\right\|_{s-1},
$$

where $\|\cdot\|_{s}$ is the usual $H^{s}\left(\mathbb{R}^{d}\right)$-norm.

Proposition 3.1 (Local existence). Denote by $\tau>0$ the existence time of smooth solution $S(t, x)$ to (3.1), and let the Assumption 1 hold. Consider the Schrödinger equation (1.5) subject to initial data, which satisfy Assumption 2 such that $U_{\text {in }}^{\epsilon} \in$ $H^{s}\left(\mathbb{R}^{d}\right)$ and $|x| U_{\text {in }}^{\epsilon} \in H^{s-1}\left(\mathbb{R}^{d}\right)$, for $s>2+d / 2$. Then there exists a time $T_{\epsilon} \in(0, \tau)$, and a unique solution to (1.5) of the following form

$$
\psi^{\epsilon}(t, x)=a^{\epsilon}(t, x) \mathrm{e}^{\mathrm{i} \Phi^{\epsilon}(t, x) / \epsilon}, \quad \text { for } 0 \leq t \leq T_{\epsilon} .
$$

Moreover, it holds

$$
U^{\epsilon} \in L^{\infty}\left(\left(0, T_{\epsilon}\right], H^{s}\left(\mathbb{R}^{d}\right)\right), \quad|x| U^{\epsilon} \in L^{\infty}\left(\left(0, T_{\epsilon}\right], H^{s-1}\left(\mathbb{R}^{d}\right)\right) .
$$

We thus know that locally-in-time the oscillatory structure of the modified WKB representation for solutions to (1.5) persists, as long as the classical rotational HJ equation has smooth solutions.

Proof. Introducing the velocities $v^{\epsilon}:=\nabla_{x} \varphi^{\epsilon}$ and $w:=\nabla_{x} S-\Omega x^{\perp}$ in (3.4), we have

$$
\left\{\begin{array}{l}
\partial_{t} v^{\epsilon}+\left(v^{\epsilon}+w\right) \cdot \nabla_{x} v^{\epsilon}+\left(\nabla_{x} w\right) v^{\epsilon}+\nabla_{x} f\left(\left|a^{\epsilon}\right|^{2}\right)=0, \\
\partial_{t} a^{\epsilon}+\left(v^{\epsilon}+w\right) \cdot \nabla_{x} a^{\epsilon}+\frac{a^{\epsilon}}{2} \nabla_{x} \cdot\left(w+v^{\epsilon}\right)=\frac{\mathrm{i} \epsilon}{2} \Delta_{x} a^{\epsilon} .
\end{array}\right.
$$

From Lemma 3.1 we know that $w=\nabla_{x} S-\Omega x^{\perp}$ is indeed sub-linear. We further separate $a^{\epsilon}$ into its real and imaginary part, i.e. $a^{\epsilon}=\alpha^{\epsilon}+\mathrm{i} \beta^{\epsilon}$, to obtain the following hyperbolic system

$$
\partial_{t} U^{\epsilon}+\sum_{j=1}^{d}\left(A_{j}\left(U^{\epsilon}\right)+B_{j}(w)\right) \partial_{x_{j}} U^{\epsilon}+M\left(\nabla_{x} w\right) U^{\epsilon}=\frac{\epsilon}{2} L U^{\epsilon},
$$

where $U^{\epsilon}=\left(\alpha^{\epsilon}, \beta^{\epsilon}, v_{1}^{\epsilon}, \cdots, v_{d}^{\epsilon}\right)^{\top}$. The coefficients matrices are

$$
\sum_{j=1}^{d} A_{j} \xi_{j}=\left(\begin{array}{ccc}
v^{\epsilon} \cdot \xi & 0 & \frac{\alpha^{\epsilon}}{2} \xi^{\top} \\
0 & v^{\epsilon} \cdot \xi & \frac{\beta^{\epsilon}}{2} \xi^{\top} \\
2 f^{\prime} \alpha^{\epsilon} \xi & 2 f^{\prime} \beta^{\epsilon} \xi & v^{\epsilon} \cdot \xi \mathbf{I}_{d}
\end{array}\right), \quad \sum_{j=1}^{d} B_{j} \xi_{j}=w \cdot \xi \mathbf{I}_{d+2}
$$


with $f^{\prime}=f^{\prime}\left(\left|a^{\epsilon}\right|^{2}+\left|b^{\epsilon}\right|^{2}\right)$ and

$$
M\left(\nabla_{x} w\right)=\left(\begin{array}{ccc}
\frac{1}{2} \nabla_{x} \cdot w & 0 & 0 \\
0 & \frac{1}{2} \nabla_{x} \cdot w & 0 \\
0 & 0 & \nabla_{x} w
\end{array}\right), \quad L=\left(\begin{array}{ccc}
0 & -\Delta & 0 \\
\Delta & 0 & 0 \\
0 & 0 & \mathbf{0}_{d}
\end{array}\right) .
$$

Observe that (3.7) can be symmetrized by

$$
Q=\left(\begin{array}{cc}
\mathbf{I}_{2} & 0 \\
0 & \frac{1}{4 f^{\prime}} \mathbf{I}_{d}
\end{array}\right)
$$

which explains the necessity for our assumption $f^{\prime}>0$. (In [3] it is shown how to overcome this difficulty in case of higher order nonlinearities.) We are thus able to proceed with energy estimates in the Sobolev space $H^{s}\left(\mathbb{R}^{d}\right)$, which follow from the classical theory for hyperbolic systems:

Lemma 3.2. Assume that $w$ is sub-linear. For $s>2+d / 2,|\alpha| \leq s$ and $|\beta| \leq s-1$, there exists a locally bounded map $\mathcal{C}(\cdot)$, satisfying $\mathcal{C}^{\prime}>0$ and $\mathcal{C}(0) \geq 1$, such that

$$
\begin{aligned}
\frac{\mathrm{d}}{\mathrm{d} t}\left\langle Q \partial_{x}^{\alpha} U^{\epsilon}, \partial_{x}^{\alpha} U^{\epsilon}\right\rangle & \leq \mathcal{C}\left(N\left[U^{\epsilon}\right]\right)\left\|U^{\epsilon}\right\|_{s}^{2}, \\
\frac{\mathrm{d}}{\mathrm{d} t}\left\langle Q \partial_{x}^{\beta}\left(x_{j} U^{\epsilon}\right), \partial_{x}^{\beta}\left(x_{j} U^{\epsilon}\right)\right\rangle & \leq \mathcal{C}\left(N\left[U^{\epsilon}\right]\right)\left(\left\|U^{\epsilon}\right\|_{s}^{2}+\left\||x| U^{\epsilon}\right\|_{s-1}^{2}\right),
\end{aligned}
$$

where $\langle\cdot, \cdot\rangle$ denotes the usual scalar product on $L^{2}\left(\mathbb{R}^{d}\right)$.

Equipped with these estimates, we are able to conclude the local-in-time existence result. Set

$$
\mathcal{E}\left[U^{\epsilon}\right]:=\sum_{|\alpha| \leq s}\left\langle Q \partial_{x}^{\alpha} U^{\epsilon}, \partial_{x}^{\alpha} U^{\epsilon}\right\rangle+\sum_{j=1}^{d} \sum_{|\beta| \leq s-1}\left\langle Q \partial_{x}^{\beta}\left(x_{j} U^{\epsilon}\right), \partial_{x}^{\beta}\left(x_{j} U^{\epsilon}\right)\right\rangle,
$$

then the estimates presented above yield

$$
\mathcal{E}\left[U^{\epsilon}(t)\right] \leq \mathcal{E}\left[U^{\epsilon}(0)\right]+C \int_{0}^{t} \mathcal{C}\left(\mathcal{N}\left[U^{\epsilon}(\tau)\right]\right) \mathcal{E}\left[U^{\epsilon}(\tau)\right] \mathrm{d} \tau .
$$

Invoking a Gronwall-type inequality we consequently arrive at

$$
\mathcal{E}\left[U^{\epsilon}\right](t) \leq \mathcal{E}\left[U^{\epsilon}(0)\right] \exp \left(C \int_{0}^{t} \mathcal{C}\left(\mathcal{N}\left[U^{\epsilon}\right](\tau)\right) \mathrm{d} \tau\right),
$$

which we expect to have a relaxed bound $2 \mathcal{E}(0)$ for a finite time where $\mathcal{N}\left[U^{\epsilon}\right] \leq C_{1}$ for $C_{1}>C_{0}=\mathcal{N}\left[U_{\text {in }}^{\epsilon}\right]$. Thus an existence time exists and satisfies

$$
t \leq T_{\epsilon}=\frac{\ln 2}{C \mathcal{C}\left(C_{1}\right)} .
$$

It is obvious that the smaller the initial data (measured by $C_{1}$ ), the larger the time-interval of existence. A local-in-time existence is thus established.

Remark 3.1. The local-in-time existence for solutions of the limiting system (2.8) can be proved analogously.

For $\epsilon \in(0,1]$, assume that the initial data satisfies $\mathcal{N}\left[U_{\text {in }}^{\epsilon}\right] \leq C_{0}<C$. Thus, for $\epsilon \in(0,1]$ fixed, the local existence shows that for any number $C_{1} \in\left(C_{0}, C\right)$, there exists a $T_{\epsilon}>0$ so that (2.6) has a unique classical solution satisfying $\mathcal{N}\left[U^{\epsilon}\right] \leq C_{1}$ for $t \in\left[0, T_{\epsilon}\right]$. Define

$$
T^{\epsilon}:=\sup \left\{0<T_{\epsilon} \leq \tau: \mathcal{N}\left[U^{\epsilon}\right](t) \leq C_{1}, \forall t \in\left[0, T_{\epsilon}\right]\right\} .
$$

Namely, $\left[0, T^{\epsilon}\right)$ is the maximal time-interval of existence and depends on $C_{1}$. It will be necessary to show that $\lim _{\epsilon \rightarrow 0} T^{\epsilon}>0$, which we shall prove in Corollary 3.1 . 
Theorem 3.1 (Convergence rates). Under the same assumptions as in Proposition [3.1, suppose that there exist $a_{\mathrm{in}}, \Phi_{\mathrm{in}} \in H^{s}\left(\mathbb{R}^{d}\right)$, for $s>2+d / 2$, such that

$$
\left\|a_{\mathrm{in}}^{\epsilon}-a_{\mathrm{in}}\right\|_{s}=\mathcal{O}(\epsilon) .
$$

Let $U:=\left(\operatorname{Re} a, \operatorname{Im} a, \partial_{x_{1}} \varphi, \ldots, \partial_{x_{d}} \varphi\right)^{\top}$ be the smooth solution to (2.6) corresponding to the initial data $\left(\Phi_{\mathrm{in}}, a_{\mathrm{in}}\right)$. If

$$
U \in L^{\infty}\left(\left(0, T^{*}\right], H^{s}\left(\mathbb{R}^{d}\right)\right), \quad|x| U \in L^{\infty}\left(\left(0, T^{*}\right], H^{s-1}\left(\mathbb{R}^{d}\right)\right)
$$

with $T^{*}>0$ finite, then there exists $\epsilon_{0}$ and $C_{*}>0$, such that for $\epsilon \leq \epsilon_{0}$

$$
\left\|a^{\epsilon}(t)-a(t)\right\|_{s} \leq C_{*} \epsilon, \quad\left\|\Phi^{\epsilon}(t)-\Phi(t)\right\|_{s} \leq C_{*} \epsilon t,
$$

for all $t \in\left[0, \min \left\{T^{*}, T^{\epsilon}\right\}\right)$.

As a consequence, we infer that the pair $(\rho, \mathbf{v})=\left(|a|^{2}, \nabla_{x} \Phi\right)$, solves the hydrodynamical system (1.7). Note that $\Phi=\varphi+S$, where $S$ is determined by the HJ equation (2.7).

Proof. As $U^{\epsilon}$ solves the forced hyperbolic system (3.7), then the corresponding limiting function $U$ is governed by

$$
\partial_{t} U+\sum_{j=1}^{d}\left(A_{j}(U)+B_{j}(w)\right) \partial_{x_{j}} U+M\left(\nabla_{x} w\right) U=0 .
$$

Denote $W^{\epsilon}:=U^{\epsilon}-U$, we thus have $L U^{\epsilon}=L W^{\epsilon}+L U$ and $W^{\epsilon}$ solves

$$
\partial_{t} W^{\epsilon}+\sum_{j=1}^{d}\left(A_{j}\left(U^{\epsilon}\right)+B_{j}(w)\right) \partial_{x_{j}} W^{\epsilon}+M\left(\nabla_{x} w\right) W^{\epsilon}=\frac{\epsilon}{2} L W^{\epsilon}+R^{\epsilon},
$$

where

$$
R^{\epsilon}:=\sum_{j=1}^{d}\left(A_{j}(U)-A_{j}\left(U^{\epsilon}\right)\right) \partial_{x_{j}} U+\frac{\epsilon}{2} L U .
$$

Using Lemma 3.2 we obtain, for $s>2+d / 2$,

$$
\frac{\mathrm{d}}{\mathrm{d} t}\left\langle Q \partial_{x}^{\alpha} W^{\epsilon}, \partial_{x}^{\alpha} W^{\epsilon}\right\rangle \leq \mathcal{C}\left(N\left[W^{\epsilon}\right]\right)\left\|W^{\epsilon}\right\|_{s}^{2}+\left\langle Q \partial_{x}^{\alpha} R^{\epsilon}, \partial_{x}^{\alpha} W^{\epsilon}\right\rangle, \quad|\alpha| \leq s,
$$

as well as

$$
\begin{aligned}
\frac{\mathrm{d}}{\mathrm{d} t}\left\langle Q \partial_{x}^{\beta}\left(x_{j} W^{\epsilon}\right), \partial_{x}^{\beta}\left(x_{j} W^{\epsilon}\right)\right\rangle \leq & \mathcal{C}\left(N\left[W^{\epsilon}\right]\right)\left(\left\|W^{\epsilon}\right\|_{s}^{2}+\left\||x| W^{\epsilon}\right\|_{s-1}^{2}\right) \\
& +\left\langle Q \partial_{x}^{\beta}\left(x_{j} R^{\epsilon}\right), \partial_{x}^{\beta}\left(x_{j} W^{\epsilon}\right)\right\rangle
\end{aligned}
$$

for $|\beta| \leq s-1$. Now we estimate terms involving $R^{\epsilon}$ in (3.12) and (3.13). This is done only for $t \in\left[0, \min \left\{T^{*}, T^{\epsilon}\right\}\right)$, in which both $U^{\epsilon}$ and $U$ are regular with uniform bounds for $\mathcal{N}\left[U^{\epsilon}\right]$ and $\mathcal{N}[U]$.

First we have

$$
\left\langle Q \partial_{x}^{\alpha} R^{\epsilon}, \partial_{x}^{\alpha} W^{\epsilon}\right\rangle \leq C\left\|\partial_{x}^{\alpha} W^{\epsilon}\right\|_{0}\left\|\partial_{x}^{\alpha} R^{\epsilon}\right\|_{0}
$$

and

$$
\left\|\partial_{x}^{\alpha} R^{\epsilon}\right\|_{0} \leq C\left\|\partial_{x_{j}} U\right\|_{s}\left\|A_{j}\left(U^{\epsilon}\right)-A_{j}(U)\right\|_{|\alpha|}+C \epsilon\left\|\partial_{x}^{\alpha} U\right\|_{0} .
$$

Note that

$$
A_{j}\left(U^{\epsilon}\right)-A_{j}(U)=\left(W^{\epsilon}\right)_{j+2} \mathbf{I}_{d+2}+\tilde{A},
$$

where the only non-zero entries of $\tilde{A}$ are $\tilde{A}_{1, j+2}=\frac{1}{2}\left(W^{\epsilon}\right)_{1}, \tilde{A}_{2, j+2}=\frac{1}{2}\left(W^{\epsilon}\right)_{2}$ and

$$
\tilde{A}_{j+2,1}=2 f^{\prime}\left(\left|a^{\epsilon}\right|^{2}\right) a^{\epsilon}-2 f^{\prime}\left(|a|^{2}\right) a, \quad \tilde{A}_{j+2,2}=2 f^{\prime}\left(\left|a^{\epsilon}\right|^{2}\right) b^{\epsilon}-2 f^{\prime}\left(|a|^{2}\right) b .
$$


Using these relations and the boundedness of $\|U\|_{s+1}$ we conclude that

$$
\left\|A_{j}\left(U^{\epsilon}\right)-A_{j}(U)\right\|_{|\alpha|} \leq C\left(\mathcal{N}\left[W^{\epsilon}\right],\|U\|_{s+1}\right)\left\|W^{\epsilon}\right\|_{s} .
$$

Therefore

$$
\sum_{|\alpha| \leq s}\left\langle Q \partial_{x}^{\alpha} R^{\epsilon}, \partial_{x}^{\alpha} W^{\epsilon}\right\rangle \leq C\left(\mathcal{N}\left[W^{\epsilon}\right],\|U\|_{s+1}\right)\left\|W^{\epsilon}\right\|_{s}^{2}+C \epsilon\|U\|_{s}\left\|W^{\epsilon}\right\|_{s} .
$$

Further calculations give

$$
\begin{gathered}
\sum_{|\beta| \leq s-1}\left\langle Q \partial_{x}^{\beta}\left(x_{j} R^{\epsilon}\right), \partial_{x}^{\beta}\left(x_{j} W^{\epsilon}\right)\right\rangle \leq \\
\left.+C \epsilon\left\|\mid \mathcal{C}\left[W^{\epsilon}\right],\right\| U \|_{s}\right)\left(\left\|W^{\epsilon}\right\|_{s-1}\left\|_{s}^{2}+\right\||x| W^{\epsilon} \|_{s-1}^{2}\right) \\
+
\end{gathered}
$$

Substituting all these estimates into (3.12) and (3.13) and integrating over $\mathbb{R}^{d}$ we obtain

$$
\mathcal{E}\left[W^{\epsilon}(t)\right] \leq \mathcal{E}\left[W^{\epsilon}(0)\right]+C \int_{0}^{t} \mathcal{C}\left(\mathcal{N}\left[W^{\epsilon}\right],\|U\|_{s+1}\right) \mathcal{E}\left[W^{\epsilon}(\tau)\right] \mathrm{d} \tau+C \epsilon \int_{0}^{t} \mathcal{N}\left[W^{\epsilon}(\tau)\right] \mathrm{d} \tau,
$$

where we have used the fact that $\mathcal{E}\left[W^{\epsilon}\right]$ and $\mathcal{N}^{2}\left[W^{\epsilon}\right]$ are equivalent in the sense that there exists a constant $C>0$ such that $C^{-1} \mathcal{N}^{2}\left[W^{\epsilon}\right] \leq \mathcal{E}\left[W^{\epsilon}\right] \leq C \mathcal{N}^{2}\left[W^{\epsilon}\right]$. Note that $\mathcal{E}\left[W^{\epsilon}(0)\right]=\mathcal{O}\left(\epsilon^{2}\right)$, we thus have for $t \in\left[0, T^{*} \wedge T\right]$

$$
\mathcal{E}\left[W^{\epsilon}(t)\right] \leq C\left(T^{*}\right) \epsilon^{2}+c \int_{0}^{t} \mathcal{C}\left(\mathcal{N}\left[W^{\epsilon}\right],\|U\|_{s+1}\right) \mathcal{E}\left[W^{\epsilon}(\tau] \mathrm{d} \tau .\right.
$$

We apply Gronwall's inequality to obtain

$$
\mathcal{E}\left[W^{\epsilon}(t)\right] \leq C \epsilon^{2} \exp \left(c \int_{0}^{t} \mathcal{C}\left(\mathcal{N}\left[W^{\epsilon}\right],\|U\|_{s+1}\right) \mathcal{E}\left[W^{\epsilon}(\tau)\right] \mathrm{d} \tau\right)=: \mathcal{F}(t) .
$$

Thus, we infer

$$
\frac{\mathrm{d}}{\mathrm{d} t} \mathcal{F}(t) \leq C \mathcal{C}\left(\mathcal{N}\left[W^{\epsilon}\right](t),\|U\|_{s+1}(t)\right) \mathcal{F}(t)^{2}, \quad \mathcal{F}(0)=C \epsilon^{2} .
$$

This differential inequality with $\mathcal{O}\left(\epsilon^{2}\right)$ initial data ensures that for fixed $T^{*}$, there exists $\epsilon_{0}$ and $C^{*}$ such that for $t \in\left[0, T^{*}\right]$ and $\epsilon \leq \epsilon_{0}$ we have

$$
\mathcal{E}\left[W^{\epsilon}(t)\right] \leq \mathcal{F}(t) \leq\left(C^{*} \epsilon\right)^{2}
$$

This leads to

$$
\left\|a^{\epsilon}(t)-a(t)\right\|_{s}=O(\epsilon), \quad\left\|\nabla_{x} \Phi^{\epsilon}(t)-\nabla_{x} \Phi(t)\right\|_{s}=O(\epsilon) .
$$

The second estimate combined with the equations for $\Phi^{\epsilon}$ and $\Phi$ yields

$$
\left\|\Phi^{\epsilon}(t)-\Phi(t)\right\|_{s}=O(\epsilon) t
$$

and the proof is complete.

Theorem 3.1 yields an approximation of $\psi^{\epsilon}$ for small times only [10], since

$$
\begin{aligned}
\left\|\psi^{\epsilon}(t)-a(t) \mathrm{e}^{\mathrm{i} \Phi(t) / \epsilon}\right\|_{L^{2}} & =\left\|a^{\epsilon}(t) \mathrm{e}^{\mathrm{i} \Phi^{\epsilon}(t) / \epsilon}-a(t) \mathrm{e}^{\mathrm{i} \Phi(t) / \epsilon}\right\|_{L^{2}} \\
& \leq\left\|a^{\epsilon}(t)-a(t)\right\|_{L^{2}}+\left\|\mathrm{e}^{\mathrm{i} \Phi(t) / \epsilon}-\mathrm{e}^{\mathrm{i} \Phi(t) / \epsilon}\right\|_{L^{\infty}}\|a(t)\|_{L^{2}} \\
& \leq \mathcal{O}(\epsilon)+\mathcal{O}(t) .
\end{aligned}
$$

In other words, to accurately approximate the wave function $\psi^{\epsilon}$ itself one has to take into account higher order corrections. Indeed, it has been shown in [10] that

$$
\left\|\psi^{\epsilon}(t)-a(t) \mathrm{e}^{\mathrm{i} \Phi_{1}(t)} \mathrm{e}^{\mathrm{i} \Phi(t) / \epsilon}\right\|_{L^{\infty}\left(\left(0, T^{*}\right] ; L^{2}\left(\mathbb{R}^{d}\right)\right)} \leq \mathcal{O}(\epsilon),
$$

where $\Phi_{1}$ is the first corrector appearing in the asymptotic expansion (2.2). The slowly varying, phase $\Phi_{1}(t, x)$, for $t \in\left[0, T^{*}\right]$, is obtained from a linear hyperbolic 
system with source terms (and thus will always be generated during the course of time). Consequently one might consider $\mathrm{a}:=a \mathrm{e}^{\mathrm{i} \Phi_{1}}$ as a new (complex-valued) WKB amplitude. Since in the present work we are mainly interested in deriving (1.7), we shall not go into further details and rather refer to [10, 3. Note however, that in the case where one aims to accurately describe the semi-classical dynamics of vortex states (see Remark 2.1) it is crucial to take into account this additional slowly varying phase $\Phi_{1}$. Even though the fluid velocity in the classical limit is given by $\nabla_{x} \Phi$ as usually, the total phase of the wave function in this asymptotic regime is $\Phi+\epsilon \Phi_{1}$. This $\mathcal{O}(\epsilon)$-correction of the phase is usually ignored in the physics literature. However, by doing so one can no longer justify a semi-classical approximation in the sense of (3.14).

With the above given result in hand, we are now able to show global-in-time convergence of the semi-classical limit.

Corollary 3.1 (Global convergence). Under the same assumptions as before and for any $C_{1}$ satisfying

$$
\mathcal{N}\left[U_{0}^{\epsilon}\right] \leq C_{0}<C_{1}, \quad \mathcal{N}\left[U^{\epsilon}(t)\right] \leq C_{1}<C, \quad \text { for } t \in\left[0, \min \left\{T^{*}, T^{\epsilon}\right\}\right),
$$

it holds $T^{\epsilon}\left(C_{1}\right)>T^{*}$ for $\epsilon>0$ sufficiently small.

In case the hydrodynamic system (1.7) is proved to admit global solution, i.e. $T^{*}=\infty$, we have consequently established convergence of solutions of (2.5) towards solutions of (1.7) globally in time.

Proof. Assume the contrary of what we aim to prove, i.e. assume that there is a $C_{1}$ satisfying (3.15) and a sequence $\epsilon_{n} \rightarrow 0$ as $n \rightarrow \infty$ and $T^{\epsilon_{n}}\left(C_{1}\right) \leq T^{*}$. Then there exists $\tilde{C}$ satisfying

$$
\mathcal{N}[U(t)]<\tilde{C}<C_{1} .
$$

From Theorem 3.1 it follows that

$$
\mathcal{N}\left[U^{\epsilon}(t)-U(t)\right] \leq C_{*} \epsilon .
$$

Thus, there is a $n \in \mathbb{N}$ such that $\mathcal{N}\left[U^{\epsilon_{n}}(t)\right] \leq \tilde{C}$ for $t \in\left[0, T^{\epsilon_{n}}\right)$. On the other hand, we have

$$
\mathcal{N}\left[U^{\epsilon_{n}}(t)\right] \leq \mathcal{N}\left[U^{\epsilon_{n}}(t)-U(t)\right]+\mathcal{N}[U(t)] \leq C_{*} \epsilon_{0}+\tilde{C}, \quad t \in\left[0, T^{\epsilon_{n}}\right) .
$$

The uniform bound of $\mathcal{N}\left[U^{\epsilon_{n}}(t)\right]$ enables us to apply the local existence result again to extend the solution beyond $T^{\epsilon_{n}}$. This contradicts the definition of $T^{\epsilon_{n}}\left(C_{1}\right)$. Thus the proof is complete.

Remark 3.2. Corollary 3.1 is an extension of the corresponding theorem in [21.

\section{Rotational DYNAMiCs OF SEMI-Classical SUPERFLUidS}

The study of superfluid dynamics in response to a rotational forcing has been the subject of vast experimental and theoretical work in the past years. The expectation value of the angular momentum, i.e.

$$
m^{\epsilon}(t):=\mathrm{i} \epsilon \int_{\mathbb{R}^{d}} \overline{\psi^{\epsilon}}(t, x) x^{\perp} \cdot \nabla_{x} \psi^{\epsilon}(t, x) \mathrm{d} x,
$$

has been mainly used to describe the dynamics [4. In particular, a nonzero value of $m^{\epsilon}(t)$ signifies the vortex nucleation in BEC experiments. For a condensate wave 
function of the form $\psi^{\epsilon}(t, x)=a^{\epsilon}(t, x) \mathrm{e}^{\mathrm{i} \Phi^{\epsilon}(t, x) / \epsilon}$ we obtain

$$
\begin{aligned}
m^{\epsilon}(t) & =-\int_{\mathbb{R}^{d}}\left|a^{\epsilon}\right|^{2} x^{\perp} \cdot \nabla_{x} \Phi^{\epsilon}(t, x) \mathrm{d} x+\mathrm{i} \epsilon \int_{\mathbb{R}^{d}} \bar{a}^{\epsilon} x^{\perp} \cdot \nabla_{x} a^{\epsilon} \mathrm{d} x \\
& =-\int_{\mathbb{R}^{d}}|a|^{2} x^{\perp} \cdot \nabla_{x} \Phi(t, x) \mathrm{d} x+\mathcal{O}(\epsilon) .
\end{aligned}
$$

In the following we denote the leading order angular momentum by

$$
m(t):=-\int_{\mathbb{R}^{d}} \rho(t, x) x^{\perp} \cdot \mathbf{v}(t, x) \mathrm{d} x,
$$

where, as before, $(\rho, \mathbf{v})=\left(|a|^{2}, \nabla_{x} \Phi\right)$. We shall also use the notation

$$
\langle g\rangle_{\rho(t)}:=\int_{\mathbb{R}^{d}} g(x) \rho(t, x) \mathrm{d} x,
$$

for any smooth function $g(x)$.

Corollary 4.1. Let $f(z)=z$ and impose the same assumptions as before. Then, as $\epsilon \rightarrow 0$ it holds

$$
m^{\epsilon}(t)=m(0)+\frac{\Omega}{2}\left(\left\langle|x|^{2}\right\rangle_{\rho(t)}-\left\langle|x|^{2}\right\rangle_{\rho_{\text {in }}}\right)+\mathcal{O}(\epsilon) .
$$

Moreover we have

$$
\frac{\mathrm{d}}{\mathrm{d} t} m^{\epsilon}(t)=\Omega\langle x \cdot \mathbf{v}\rangle_{\rho(t)}+\frac{\delta}{2 \omega_{\perp}^{2}}\left\langle x_{1} x_{2}\right\rangle_{\rho(t)}+\mathcal{O}(\epsilon),
$$

where $\delta=\frac{\omega_{1}^{2}-\omega_{2}^{2}}{\omega_{1}^{2}+\omega_{2}^{2}}$ denotes the trap deformation and $\omega_{\perp}^{2}=\frac{1}{2}\left(\omega_{1}^{2}+\omega_{2}^{2}\right)$ the radial frequency.

In the semi-classical regime, the angular momentum expectation value is dominated by the classical rotational effect, in contrast to the results of [4, 6] (where the GPE is considered unscaled). The result also shows that a non-isotropic frequency for the trapping potential contributes to the change of the angular momentum.

Proof. Let

and we also introduce

$$
n(t):=\int_{\mathbb{R}^{d}} \rho(t, x) x \cdot \mathbf{v}(t, x) \mathrm{d} x
$$

$$
X^{\epsilon}(t):=\int_{\mathbb{R}^{d}}|x|^{2}\left|\psi^{\epsilon}(t, x)\right|^{2} \mathrm{~d} x=\left\langle|x|^{2}\right\rangle_{\rho(t)}+\mathcal{O}(\epsilon),
$$

A straightforward calculation calculation then yields

$$
\frac{\mathrm{d}}{\mathrm{d} t} m(t)=\Omega n(t)+\int_{\mathbb{R}^{d}} \rho\left(x^{\perp} \cdot \nabla_{x}\right) V \mathrm{~d} x .
$$

When combined with a quadratic potential of the form $V=\frac{1}{2}|\omega \cdot x|^{2}$, this leads to the relation (4.4). Also, one easily verifies that

$$
\frac{\mathrm{d}}{\mathrm{d} t}\left\langle|x|^{2}\right\rangle_{\rho(t)}=2 n(t)
$$

which, upon inserting into (4.5), yields (4.3) after an integration w.r.t. time.

We also remark that the change of $n(t)$ also depends on $m(t)$ as well as the total energy. This can be seen as follows: First we calculate

$$
\frac{\mathrm{d}}{\mathrm{d} t} n(t)=-\int_{\mathbb{R}^{d}} \rho\left(x \cdot \nabla_{x}\right)(V(x)+f(\rho)) \mathrm{d} x+\int_{\mathbb{R}^{d}} \rho \mathbf{v}^{2} \mathrm{~d} x
$$


and an integration by parts gives

$$
\frac{\mathrm{d}}{\mathrm{d} t} n(t)=\int_{\mathbb{R}^{d}}\left(\rho^{2}+\rho \mathbf{v}^{2}\right) \mathrm{d} x-\int_{\mathbb{R}^{d}} \rho\left(x \cdot \nabla_{x}\right) V(x) \mathrm{d} x .
$$

Note that for $f(z)=z$, the total energy (1.6) expressed in terms of $a$ and $\mathbf{v}$ reads

$$
E=\int_{\mathbb{R}^{d}} \frac{1}{2} \rho\left|\mathbf{v}-\Omega x^{\perp}\right|^{2}+\rho\left(V-\frac{\Omega^{2}}{2}\left(x_{1}^{2}+x_{2}^{2}\right)\right)+\frac{1}{2} \rho^{2} \mathrm{~d} x+\mathcal{O}(\epsilon) .
$$

For quadratic potentials $V=\frac{1}{2}|\omega \cdot x|^{2}$, it holds $\left(x \cdot \nabla_{x}\right) V(x)=2 V(x)$, which consequently implies

$$
\begin{aligned}
\frac{\mathrm{d}}{\mathrm{d} t} n(t) & =2\left(E-\Omega m(t)-\int_{\mathbb{R}^{d}} \rho V(x) \mathrm{d} x\right)-\int_{\mathbb{R}^{d}} \rho\left(x \cdot \nabla_{x}\right) V(x) \mathrm{d} x \\
& =2(E-\Omega m(t))-4 \int_{\mathbb{R}^{d}} \rho V(x) \mathrm{d} x .
\end{aligned}
$$

Combining these calculations yields the following closed system

$$
\left\{\begin{array}{l}
\frac{\mathrm{d}}{\mathrm{d} t} n(t)=2(E(0)-\Omega m(t))-2 \int_{\mathbb{R}^{d}}|\omega \cdot x|^{2} \rho \mathrm{d} x, \\
\frac{\mathrm{d}}{\mathrm{d} t} m(t)=\Omega n(t)+\left(\omega_{x_{1}}^{2}-\omega_{x_{2}}^{2}\right) \int_{\mathbb{R}^{d}} x_{1} x_{2} \rho \mathrm{d} x
\end{array}\right.
$$

Finally, we note that for an isotropic harmonic confinement, i.e. $V=\frac{1}{2} \omega^{2}|x|^{2}$, we have

$$
\frac{\mathrm{d}}{\mathrm{d} t} m(t)-\Omega n(t)=0, \quad \frac{\mathrm{d}}{\mathrm{d} t} n(t)+2 \Omega m(t)=2 E(0)-2 \omega^{2}\left\langle|x|^{2}\right\rangle_{\rho(t)} .
$$

Since in this case, it also holds

$$
\frac{\mathrm{d}}{\mathrm{d} t} X=2 n(t)=\frac{2}{\Omega} \frac{\mathrm{d}}{\mathrm{d} t} m(t)
$$

and we simply obtain

$$
m(t)=\frac{\Omega}{2} X(t)+m(0)-\frac{\Omega}{2}\left\langle|x|^{2}\right\rangle_{\rho_{\text {in }}} .
$$

The obtained relations yield a closed equation for $m(t)$, i.e.

$$
\frac{\mathrm{d}^{2}}{\mathrm{~d} t^{2}} m(t)+\left(2 \Omega^{2}+4 \omega^{2}\right) m(t)=2 \Omega E(0)+4 m(0) \omega^{2}-2 \Omega \omega^{2}\left\langle|x|^{2}\right\rangle_{\rho_{\text {in }}} .
$$

The general solution of it can be written as

$m=C_{1} \cos \sqrt{4 \omega^{2}+2 \Omega^{2}} t+C_{2} \sin \sqrt{4 \omega^{2}+2 \Omega^{2}} t+\frac{\Omega E(0)+\Omega \omega^{2}\left(2 m(0)-\left\langle|x|^{2}\right\rangle_{\rho_{\text {in }}}\right)}{\Omega^{2}+2 \omega^{2}}$,

where $C_{1}$ and $C_{2}$ are determined by $m(0)$ and $\left.\frac{\mathrm{d}}{\mathrm{d} t} m(t)\right|_{t=0}=\Omega n(0)$. The above given calculations could be used to compare numerical simulations of the full NLS dynamics in the spirit of [4, 6].

Acknowledgment. The authors thank R. Carles for helpful discussions. H. Liu wants to thank the WPI (Vienna) for its hospitality and support during his visit in May 2006 when this work was initiated. 


\section{REFERENCES}

[1] A. Aftalion, Properties of vortices in Bose-Einstein condensates, C.R. Physique 5 (2004), $9-20$.

[2] A. Aftalion and Q. Du, Vortices in a rotating Bose-Einstein condensate: critical angular values and energy diagrams in the Thomas-Fermi regime, Phys. Rev. A 64 (2001), 063603.

[3] T. Alazard and R. Carles, Supercritical geometric optics for nonlinear Schrödinger equations, preprint arXiv: 07042488.

[4] W. Bao, Q. Du, and Y. Zhang, Dynamics of rotating Bose-Einstein condenstates and its efficient and accurate numerical computation, SIAM J. Appl. Math. 66(2006), no. 3, 758-786.

[5] W. Bao, D. Jaksch and P.Markowich, Numerical solution of the Gross-Pitaevskii Equation for Bose-Einstein condensation, J. Comput. Phys. 187(2003), no.1, 318-342.

[6] W. Bao, D. Jaksch A. Klein, and Y. Zhang, Dynamics of vortices in weakly interacting BoseEinstein condensates, preprint 2007.

[7] W. Bao, F. Y. Lim, and Y. Zhang, Energy and chemical potential asymptotics for the ground state of Bose-Einstein condensates in the semi-classical regime, Bull. Inst. Math. Acad. Sinica 2 (2007), no. 2, 495-532.

[8] W. Bao, P. Markowich, and H. Wang, Ground, symmetric and central vortex states in rotating Bose-Einstein condensates, Comm. Math. Sci. 3 (2005), no. 1, 57-88.

[9] W. Bao and H. Wang, An efficient and spectrally accurate numerical method for computing dynamics of rotating Bose-Einstein condensates, J. Comput. Phys. 217 (2006), no. 2, 612-626.

[10] R. Carles, WKB analysis for nonlinear Schrödinger equations with potential. Comm. Math. Phys. 269 (2007), no. 1, 195-221.

[11] R. Carles, On the semi-classical limit for the nonlinear Schrödinger equation, preprint arXiv: math/0612518v1.

[12] Y. Castin and R. Dum, Bose-Einstein condensates with vortices in rotating traps, The European Physical Journal D 7 (1999), no.3, 399-412.

[13] M. Correggi, T. Rindler-Daller, and J. Yngvason, Rapidly Rotating Bose-Einstein Condensates in Strongly Anharmonic Traps, J. Math. Phys. 48 (2007), 042104-42134.

[14] B. Desjardins, C. K. Lin, and T. C. Tso, Semiclassical limit of the derivative nonlinear Schrödinger equation, Math. Mod. Meth. Appl. Sci. 10 (2000), no. 2, 261-285.

[15] P. Rosenbusch, V. Bretin, and J. Dalibard, Dynamics of a Single Vortex Line in a Bose-Einstein Condensate, Phys. Rev. Lett. 89 (2002), 200403.

[16] R. J. Donnelly, Quantized vortices in Helium II, Cambrige University Press 1995.

[17] L. Erdös, B. Schlein, and H.-T. Yau, Derivation of the cubic non-linear Schrödinger equation from quantum dynamics of many-body systems, Invent. Math. 167 (2007), no. 3, 515-614

[18] I. Gasser, C. K. Lin, and P. A. Markowich, A Review of Dispersive Limits of (Non)Linear Schrödinger Type Equations, Taiwanese Journal of Mathematics 4 (2000), 501-529.

[19] E. Grenier, Semiclassical limit of the nonlinear Schrödinger equation in small time, Proc. Am. Math. Soc. 126 (1998), no. 2, 523-530.

[20] H. Liu, Critical thresholds in the semi-classical limit of 2-D rotational Schrödinger equations, Z. angew. Math. Phys. 57 (2006), 42-58.

[21] H. Liu and E. Tadmor, Semiclasscial limit of the nonlinear Schrödinger-Poisson equation with subcritical initial data, Methods and Applications of Analysis 9(4) (2002), 517-531.

[22] L. Pitaevskii and S. Stringari, Bose-Einstein condensation, Internat. Series of Monographs on Physics 116, Clarendon Press, Oxford (2003).

[23] A. Recati, F. Zambelli, and S. Stringari, Overcritical rotation of a trapped Bose-Einstein condensate, Phys. Rev. Lett. 86 (2001), 377-380.

[24] F. Zambelli and S. Stringari, Moment of inertia and quadrupole response of a trapped superfluid, Phys. Rev. A 63 (2001), 33602.

Department of Mathematics, Iowa State University, Ames, IA 50011-2064

E-mail address: hliu@iastate.edu

Wolfgang Pauli Institute Vienna, Nordbergstrasse 15, A-1090 Vienna, Austria and DamtP, Centre for Mathematical Sciences, Wilberforce Road, Cambridge CB3 0WA, UK.

E-mail address: christof.sparber@univie.ac.at 